$\begin{array}{ll}\text { le portiQue } & \text { Le Portique } \\ \text { Revue de philosophie et de sciences humaines }\end{array}$

2| 1998

\title{
Freud et la philosophie
}

\section{L'angoisse paroxysmale}

\section{Antoine Vergote}

\section{(2) OpenEdition}

\section{Journals}

\section{Édition électronique}

URL : http://journals.openedition.org/leportique/340

DOI : 10.4000/leportique.340

ISSN : $1777-5280$

\section{Éditeur}

Association "Les Amis du Portique"

\section{Édition imprimée}

Date de publication : 1 septembre 1998

ISSN : 1283-8594

\section{Référence électronique}

Antoine Vergote, "L'angoisse paroxysmale », Le Portique [En ligne], 2 | 1998, mis en ligne le 15 mars 2005, consulté le 26 mars 2021. URL : http://journals.openedition.org/leportique/340 ; DOI : https:// doi.org/10.4000/leportique.340

Ce document a été généré automatiquement le 26 mars 2021.

Tous droits réservés 


\title{
L'angoisse paroxysmale
}

\author{
Antoine Vergote
}

1 En choisissant cet objet pour réflexion je voudrais tout d'abord prendre part à la recherche proposée comme thème majeur de notre présent congrès: l'analyse szondienne de la paroxysmalité ${ }^{1}$. Mais au-delà de l'attention clinique au phénomène de la paroxysmalité, mon étude voudrait aussi contribuer à l'interrogation, explicite - ou sous-jacente, qui représente toujours l'intérêt spécifique des congrès de l'analyse du destin : l'interrogation du lien entre Szondi et Freud, plus particulièrement, celle du projet szondien de fournir la théorie globale des pulsions que Freud n'aurait pas pu ou pas voulu - élaborer. Centré sur un phénomène particulier, mon exposé n'a pas la prétention de donner une réponse englobante aux vastes questions qu'on vient d'énoncer. Il est néanmoins légitime d'attendre d'une analyse un peu poussée d'un fait clinique singulier qu'elle fournisse des indications sur les principes qui sont en jeu dans les discussions des rapports entre Szondi et Freud.

2 Ainsi que le suggère le terme formé avec le mot grec Pan, l'angoisse panique envahit le sujet tout entier: pensée, affectivité, corps. En appelant pareille angoisse " paroxysmale », Szondi y désigne, premièrement, la décharge violente de "l'affect ", « pulsion paroxysmale » elle-même violente ; deuxièmement, l'expression corporelle de cette décharge qui a les caractéristiques de la crise hystérique, elle aussi relevant du vecteur paroxysmal.

3 Je me permets de vous faire d'abord part d'une impression que m'ont fait des crises d'angoisse paroxysmale, car cette impression fait partie des impondérables qui orientent l'écoute et les interventions du clinicien. En assistant à de telles crises d'angoisse au cours de mes activités cliniques, j'ai été frappé par certaines similitudes avec les transes des possessions rituelles dont j'ai été témoin. Dans les deux cas, le sujet se trouve dans un état second apparemment semblable et donne à voir des manifestations corporelles similaires. Dans l'angoisse paroxysmale, le patient paraît bien être l'enjeu d'un théâtre de possession ; autrement dit, dans les deux sens du mot, il est le "sujet » de forces qui jouent en lui et avec lui et au jeu cruel desquelles il se prête involontairement. Toutefois ces deux phénomènes se caractérisent par une différence qui est significative à l'intérieur de leur parenté apparente : l'inversion du 
rapport entre les tonalités affectives. Le patient éprouve l'angoisse paroxysmale avec un déplaisir manifeste, tout en y étant attaché de manière secrète et ambiguë, ainsi que l'attestent indéniablement les associations produites au cours de l'analyse. En revanche les sujets qui ont part aux possessions rituelles donnent à voir un ravissement auquel se mêle visiblement une tension douloureuse. Ces émotions différentes correspondent à des contenus représentatifs différents. Des représentations mythologiques régissent les véritables possessions rituelles et déterminent les règles à suivre pour réussir celles-ci. Dans les crises d'angoisse éclatent des représentations inconscientes et, dans l'ambivalence affective de ces expériences, faites d'angoisse et d'attachement sousjacent, on peut reconnaitre la caractéristique du symptôme psychopathologique tel que Freud en a analysé la structure.

4 Cette caractérisation de l'angoisse paroxysmale n'est encore qu'intuitive. Elle contient néanmoins déjà plusieurs questions que se pose le clinicien ayant quelque expérience de pareil phénomène. L'angoisse paroxysmale est-elle également un état de possession? Une possession par l'effet de l'inconscient? Dans la pathologie, le sujet serait-il purement passif lors de la production psychologique de la possession? Et possession par quoi? L'ambivalence affective qui y transparait, de souffrance et de plaisir, aurait-elle le sens d'un symptôme appartenant à une névrose comme l'hystérie ou l'obsession? Comme de telles crises paroxysmales se produisent quelques fois au cours d'une cure analytique, les associations contextuelles permettent heureusement de formuler avec précision les questions que l'on se pose devant ces faits cliniques et de chercher à y répondre.

5 Pour cerner les faits d'observation et pour en dégager le sens, il faut évidemment un cadre théorique. Selon ma conviction, il est tout indiqué de recourir essentiellement aux conceptions théoriques de Freud et de Szondi. Ce faisant nous aurons l'occasion de comparer entre elles leurs conceptions et d'en juger en référence à l'observation clinique. L'analyse de l'angoisse paroxysmale représente même une situation privilégiée pour pareille confrontation, car elle conduit à faire intervenir des éléments essentiels de leurs théories respectives. Dès le départ de ses activités psychopathologiques Freud a reconnu dans l'angoisse une des grandes énigmes psychologiques et tout au long de ses recherches il a poursuivi les efforts pour la clarifier. Quant à Szondi, son attention particulière aux phénomènes paroxysmaux est bien connue. Un de ses mérites est même d'éclairer fortement ce phénomène que Freud n'a pas aussi explicitement considéré, en tout cas pas durant les vingt premières années des travaux au cours desquels il a élaboré l'essentiel de son système théorique. Cependant, si Szondi introduit l'idée nouvelle de pulsion paroxysmale, il est néanmoins convaincu de renouer par là avec le concept freudien tardif de pulsion de mort.

6 La première partie de mon étude proposera une brève confrontation entre les concepts de Freud et de Szondi en rapport aux cas cliniques qui nous occupent ici. Instruit par cette comparaison critique, dans la deuxième partie je présenterai mon interprétation de l'angoisse paroxysmale.

1. Angoisse et affect

7 Dès ses premières études analytiques, Freud s'astreint à comprendre l'angoisse et la pulsion l'une par rapport à l'autre. On ne peut pas se faire une idée juste de la théorie freudienne de la pulsion si on ne considère pas la première théorie freudienne de l'angoisse. Toute discussion sur les rapports théoriques entre Szondi et Freud doivent donc commencer par revenir au concept freudien fondamental de pulsion tel qu'il l'a 
établi en comprenant que la possibilité de l'angoisse lui est inhérente. Pour Freud la pulsion (Trieb, qu'il décide de distinguer d'Instinkt afin d'éviter les confusions), est la force orientée de la vie corporelle qui se fait réalité psychique par le lien avec la "représentation de désir». Freud appelle «affect» cette force de vie en tant qu'elle comporte une grandeur de poussée (Drang). Si Freud mentionne plusieurs pulsions, dans son élaboration du concept de pulsion il se réfère essentiellement à la pulsion libidinale. Ceci est également fort important pour la théorie freudienne de la pulsion; pour faire bref, je dirais que les autres pulsions (de conservation de soi, etc.) - la pulsion de mort de quelque manière exceptée - n'ont la qualité de pulsion que par leur connexion avec la libido. Selon la première théorie de Freud, l'angoisse consiste dans la déliaison (Entbindung) qui a lieu - suite au refoulement - entre l'affect pulsionnel et la représentation. Lorsqu'après l'introduction du narcissisme et l'analyse du processus du refoulement, Freud proposera une autre conception de l'angoisse, il n'abandonnera pas pour autant « la première théorie ». Cette dernière interprète en réalité adéquatement ce qui se passe essentiellement dans l'angoisse: le psychisme - je dirais: le corps psychique - se trouve envahi par l'affect devenu chaotique ; ceci précisément parce que la suppression de la représentation lui a enlevé la médiatisation pour l'investissement dans le monde extérieur qui permettrait de " décharger » l'affect. Afin de résoudre la tension qui se fait insupportable, le patient cherche souvent un objet symbolique substitutif. Dans la phobie, cet objet symbolise l'instance qui a provoqué le refoulement. Dans l'hystérie, l'investissement se fait dans une partie du corps propre « choisi » comme substitut symbolique en liaison avec la représentation médiatrice supprimée. Mais, ainsi que le suggéraient déjà les démonstrations théâtrales de Charcot, la pulsion libidinale, éclatant dans l'angoisse, peut retrouver en elle-même son investissement ambivalent. Il faudra cependant l'avancée de la théorie analytique pour comprendre pareille érotisation de l'angoisse.

Du moment où l'on essaie d'analyser le processus du refoulement lui-même, s'impose l'idée d'une sorte de peur plus ou moins inconsciente qui en est responsable. Il n'est pas étonnant, dès lors, que Freud revienne sur la question de l'angoisse, donnée plus complexe qu'il n'avait d'abord pensé. En 1926, dans Inhibition, symptôme et angoisse, Freud met effectivement en avant un autre aspect de l'angoisse, plus proche de la peur, et qu'il identifie par l'expression éloquente : Signalangst, angoisse comme signal. Ayant entre-temps élaboré la théorie du moi, Freud situe maintenant la fonction de l'angoisse dans le processus du refoulement. Le moi qui perçoit un danger, interne ou externe, fait inconsciemment revivre le souvenir d'une ancienne expérience traumatique, relie la perception du danger actuel à ce souvenir revivifié, et produit ainsi l'angoisse comme sentiment qui l'affecte, qu'il subit. Le moi se met ainsi en état de se défendre psychologiquement (involontairement, bien sûr) contre le danger actuel. Par ce processus le sujet est, en effet, préparé à affronter des expériences gravement traumatisantes, alors que celui qui ne dispose pas de l'Angstbereitschaft, de la disposition défensive de l'angoisse, pourra être à ce point surpris et saisi par une expérience de danger qu'il deviendra la victime d'une "névrose traumatique», celle où se répète hallucinatoirement, comme dans un rêve éveillé, le souvenir de l'accident, de l'agression subie, de la violence destructrice perçue, ainsi que l'effroi (Schreck) éprouvé à ce moment. Cette névrose n'est même plus une des névroses où le refoulement sauvegarde une intégrité suffisante du moi et engendre une solution de compromis, celle sur laquelle pourra travailler la cure analytique. 
9 Les théories de Freud me semblent adéquatement expliquer aussi bien les angoisses qui préparent les névroses que celles qui résultent du refoulement et qui, dans leur érotisation, en portent la marque. Freud ne s'est cependant pas réellement attaché à considérer l'angoisse proprement paroxysmale. Comme il le reconnaîtra, l'immense tâche d'analyser et d'expliquer les névroses a pendant des décennies polarisé son attention sur la pulsion libidinale et sur les formations névrotiques - hystérie, phobie, obsession - produites comme solutions pour l'angoisse résultant du refoulement. Dès lors, il m'a paru indiqué de me tourner vers Szondi qui a fortement mis en profil le "vecteur paroxysmal », dans sa théorie comme dans sa pratique.

Szondi, lui aussi, explique l'angoisse par le débordement du sujet par l'affect. Ne nous y trompons cependant pas, les mêmes mots et les mêmes formulations recouvrent des théories fondamentalement différentes de part et d'autre. Pour Szondi l'affect n'est pas le concept «économique » freudien désignant la poussée de la pulsion comme telle, mais une catégorie définie des pulsions, celle qui forme essentiellement le vecteur paroxysmal. L'affect est la pulsion que Szondi définit répétitivement à travers toute son œuvre par la séquence suivante: poussée (Drang), colère, rage, jalousie, envie, vengeance, das Böse (le mal ou la méchanceté destructrice). Les cas e+ hy+ sont ceux des sujets inondés par le torrent de l'affect (Affektflut), inondation qu'accompagne l'angoisse morale (Gewissensangst). Les cas e- hy-: de panique d'affect (Affekt-Panik), également appelée : angoisse panique. Pour Szondi ces deux types représentent les deux formes de défusion pulsionnelle (Triebentmisschung) verticale de l'affect. L'affect accumulé (stauen) par la marée montante (Flut), signalé par le e- !, produit une attaque (Anfall) interne. Rappelons que, selon la première théorie de Freud, l'angoisse est une sorte d'attaque interne par l'affect ; mais Freud vise par là la poussée libidinale privée de la représentation médiatrice pour l'action. Lorsque Szondi parle de l'attaque de l'affect, il s'agit de l'affect défini comme colère, rage etc. De cette manière Szondi peut écrire qu'« une action mauvaise (böse) est, du point de vue de l'analyse du destin, une attaque paroxysmale épileptique. » (Die Triebentmisschten, Bern, Huber, 1980, p. 168).

11 La conception szondienne de la pulsion paroxysmale (rage, das Böse...) évoque évidemment des idées auxquelles Freud aboutit et qu'il rassemble dans le concept énigmatique de pulsion de mort. Un texte impressionnant que Freud écrit en 1929, dans Malaise dans la civilisation, parait conforter la théorie de la parenté entre la pulsion freudienne de mort et l'affect paroxysmal de Szondi. "Je reconnais que nous avons toujours eu devant les yeux les manifestations de la pulsion de destruction tournée vers le dehors ou vers le dedans, lorsque, dans le sadisme et le masochisme, la pulsion de destruction était fortement liée (legiert) à l'érotique. Mais je ne comprends plus que nous ayons pu méconnaître l'omniprésence des agressions et destructions nonérotiques. » (GW, XIV, p. 479). Freud avait, en effet, toujours refusé la thèse de la pulsion de mort que Sabina Spielrein avait avancée avec force.

Ici encore la similitude apparente entre les thèses de Freud et de Szondi ne devraient pas nous leurrer et nous cacher les différences essentielles. 1) Pour Freud la pulsion de mort est d'abord orientée en dedans, contre le moi tel qu'il est pris dans les représentations libidinales de désir. C'est bien en cette qualité que, selon Freud, la pulsion de mort sert de base à la négation linguistique, « symbole » très important dans la reconnaissance de la réalité. 2) Freud ne rattache pas, comme le fait Szondi, les qualifications du mal moral (das Böse) à la pulsion de mort. Tout comme la libido, elle est moralement neutre. 3) Étant précisément un principe négatif, la pulsion freudienne 
de mort n'a pas son énergie en elle-même. Aussi est-elle d'abord en l'état de fusion avec la libido et n'acquiert-elle une fonction autonome que par la défusion. Pour Szondi, en revanche, la pulsion paroxysmale est une pulsion ayant sa substance pulsionnelle en elle-même. Pour la maitriser, le moi doit en opérer la fusion avec d'autres pulsions ou avec l'autre facteur du vecteur paroxysmal, celui du hy. On l'observe à nouveau : les conceptions de la pulsion, et donc également de la défusion pulsionnelle, sont fondamentalement différentes de part et d'autre. 4) La pulsion freudienne de mort peut causer l'angoisse morale en se liant au surmoi et en lui donnant un caractère de férocité. Mais n'ayant pas son énergie pulsionnelle de par elle-même, la pulsion de mort ne saurait expliquer l'angoisse panique comme une sorte de débordement du moi par une marge pulsionnelle.

13 Laissons de côté les problèmes inextricables que pose la pulsion freudienne de mort. Ma question ici est de savoir si on peut comprendre l'angoisse paroxysmale par l'attaque interne de la pulsion paroxysmale de Szondi.

La thèse que je voudrais avancer et brièvement illustrer, consiste à interpréter l'idée szondienne de "pulsion paroxysmale " comme l'indice d'un phénomène clinique complexe, dont on occulterait la signification et l'articulation en prenant l'expression "pulsion paroxysmale» comme un concept scientifique de base, un concept qui désigne la chose elle-même qu'il évoque. À moins que l'on espère encore et malgré tout, à la suite de Szondi, donner un fondement biologique spécifique à la "pulsion paroxysmale", il faudra, selon moi, comprendre ce terme comme la condensation métaphorique d'une histoire de complexification pulsionnelle et égoïque. Dans ma conviction, ce terme n'a pas le même statut scientifique que celui de libido dans la théorie freudienne. C'est du moins la conviction à laquelle je suis parvenu après bien des hésitations, car, comme je l'ai souligné, Szondi a mis en lumière certaines données cliniques auxquelles Freud n'avait pas accordé l'importance qu'elles méritent. Je propose donc de nous mettre à explorer la complexification pulsionnelle pour laquelle l'expression szondienne de pulsion paroxysmale sert d'idée directrice, idée qui nous stimule à analyser des observations auxquelles Szondi plus que Freud a été attentif.

Sans doute sera-ce toujours par la confrontation entre les données testologiques et les observations faites au cours de cures analytiques qu'on avancera dans les questions que notre congrès met à l'ordre du jour. Pour des raisons de circonstance, en particulier la reprise en analyse après l'échec thérapeutique précédent, je ne dispose malheureusement pas du test de Szondi des trois sujets auxquels je me rapporterai spécialement.

2. Interprétation analytique de l'angoisse paroxysmale

16 Force m'est ici d'extraire, de longues histoires déployées au cours de thérapies, quelques segments et d'en tirer les interprétations par lesquelles je pense pouvoir répondre aux questions que je me suis posées en rapport à la paroxysmalité.

Ce qui m'a en tout premier lieu intrigué, c'est que certains de ces sujets relevant, en termes szondiens, du vecteur paroxysmal, ont vécu durant la puberté et la première adolescence des phases d'hypochondrie. Celles-ci étaient de toute évidence solidaires des turbulences affectives et sexuelles et des secousses que subit l'image du corps. On sait que Szondi interprète l'hypochondrie comme « un besoin de punition » consécutif à la poussée de violence paroxysmale. Dans les cas auxquels je me réfère, l'hypochondrie ne résultait pas, en tout cas, de menaces portées sur la masturbation, comme cela a pu être le cas en d'autres milieux éducatifs. Ce que j'ai entendu me fait 
même fortement douter de l'interprétation szondienne de l'hypochondrie en général, et m'incite à proposer, à titre d'hypothèse, une interprétation toute différente. Il s'agit de sujets masculins doués d'une grande sensibilité aux motions et aux émotions qui se passent dans le corps propre, des sujets aussi qui éprouvaient intensément leur participation affective et imaginative au monde, en particulier à la nature. En termes szondiens : des sujets marqués par le p-!, sûrement pendant la première adolescence. Il m'a semblé que, dans cette hypochondrie, s'est développée, sur la base des turbulences libidinales agressives et de l'intense sensibilité participative, la représentation fantasmagorique libidinale-anale d'un mal anonyme presque physique, rôdant dans le monde et dans le corps en transformation, un mal comme un cancer fantasmatique qui est à l'intérieur du corps, mais qui est aussi dans le monde et pénètre dans le corps poreux et ouvert sur le monde. Cette représentation, d'autant plus angoissante que les contours en sont indéfinis, rappelle le mal de l'impur ou de la souillure que l'anthropologie culturelle a observé dans beaucoup de cultures anciennes. On songe aussi au phénomène analogue qu'on observe régulièrement chez les schizophrènes lorsqu'ils parlent de la pourriture qui habite secrètement leur corps. Freud a d'ailleurs reconnu dans l'hypochondrie une forme psychotique de la rétractation de l'investissement libidinal sur le corps propre, toutefois sans expliquer pourquoi cette rétractation est précisément douloureuse. Bien sûr, les comparaisons n'autorisent pas à assimiler les unes aux autres ces trois figures. Reste qu'un fond affectifreprésentationnel inconscient commun s'organise rituellement dans le tabou de la souillure, s'insinue sournoisement dans les angoisses hypochondriaques et se condense en substance quasi-ontologique dans la psychose.

Ces adolescents hypochondriaques, sujets doués imaginativement - au contraire des psychosomatiques - peuvent développer, en dehors de toute éducation religieuse ou en marge de celle-ci, l'idée d'un mal plus ou moins personnifié, d'une sorte de divinité démoniaque, méchante, juge féroce, jalouse, une reproduction de Kakos théos grec. Ainsi que l'affirme Mélanie Klein, il arrive qu'en l'absence d'une vraie figure paternelle symbolique qui donne la loi, le sujet construit lui-même un surmoi persécuteur qui se fait l'allié de la divinité démoniaque.

19 Ce qui m'a frappé, c'est que, dans leurs fantasmes, ces sujets s'identifient régulièrement à cette puissance démonique. Cela se passe précisément dans les moments affectifs d'un lien participatif et libidinal intense. Ainsi la tendresse éprouvée avec plaisir pour l'enfant que l'on caresse ou avec lequel on joue est soudainement traversée par le fantasme émotionnellement chargé de détruire le petit être fragile, cela par pur plaisir de destruction. Ou bien, en caressant la femme aimée on se représente subitement, avec une intense émotion, qu'on pourrait fort bien l'étrangler. En écoutant ces récits torturant pour le patient, je n'ai vraiment pas pu percevoir des signes de sadisme et de masochisme libidinaux. Il est évidemment normal qu'on songe d'abord à cette interprétation, car elle en appelle encore à une motivation positive, quoique pervertie, celle de la libido avec son effet de plaisir. On comprend que Freud, ainsi qu'il l'avoue dans le texte cité de 1929, ait longtemps rejeté une interprétation différente et qui s'engage dans la reconnaissance de ce qui parait radicalement irrationnel: la destruction que n'anime pas un plaisir libidinal.

$20 \mathrm{Ne}$ fermons toutefois pas l'interrogation par le terme de pulsion de mort, aussi énigmatique que le fait clinique lui-même. Écoutons d'abord ce qui s'entre-dit dans les paroles en liberté d'association. Un premier trait à noter est que les patients attestent 
une forte inflation du moi, combinée avec une intense participation affective et imaginative à des puissances "perçues » comme présentes dans le cosmos : puissances libidinales, avec lesquelles on s'éprouve parfois en fusion, mais également puissances de destruction, décrites comme le pur mal. Je crois que, dans les phases antérieures d'hypochondrie, il y avait une participation indécise au mal comme un impur corrosif, et que par après, dans l'inflation par participation affective et par identification imaginaire, se développe un certain état de possession (Bessessenheit) par le mal destructeur. Cet état est très angoissant parce qu'il comporte deux graves dangers que signale précisément l'angoisse. Premièrement, le sujet s'éprouve comme perdu parce que débordé ; deuxièmement, l'alternance du bien qu'on estime et du mal qui possède inquiète profondément et prive le sujet de toute confiance en lui-même.

Il arrive alors que la lecture ou l'écoute du mot "démon » ou "diable » déclenche le fantasme d'être réellement possédé. Ce fantasme - prenant éventuellement la force d'une croyance - est tout à la fois angoissant, exaltant et déculpabilisant. On est horrifié à l'idée d'être ainsi possédé par le mal ; angoissé en imaginant la mise en œuvre de ses incitations; on s'éprouve aussi soulagé à l'idée d'être la victime d'une puissance étrangère. Mais l'état de possession exalte également par l'intensité affective qu'il fait éprouver. De cette manière cette expérience intensifie à son tour l'inflation. Le patient s'y éprouve le sujet, aux deux sens du mot, d'un drame grandiose qui l'arrache aux banalités quotidiennes de l'homme sans qualités.

Cette expérience représente le fond sur lequel se déploie la dimension hystérique de ces névroses paroxysmales. Les sujets mettent en scène pour eux-mêmes, effectivement et imaginativement, la grandeur et la violence dont ils sont la source et la victime, le sujet actif et passif. Bien sur cette mise en scène se fait involontairement, par un réfléchissement narcissique de l'ego sur ses propres sentiments.

L'attachement narcissique à l'inflation participative de teneur alternativement libidinale et mortifère, l'attachement également à l'angoisse et à sa mise en scène pour soi en des crises paroxysmales, se manifestent de manière saisissante dans le malaise qu'éprouvent ces sujets lorsque l'angoisse se tait suite à la cure analytique. L'existence leur paraît alors appauvrie de son intensité douloureuse et secrètement exaltante.

En guise de conclusion

Je me permettrai de formuler comme thèses les résultats des analyses que je viens d'esquisser :

\section{Concernant l'angoisse}

Le type de patient ici évoqué s'éprouve de toute évidence en danger et l'angoisse a certainement chez lui la fonction de signal. Toutefois, il ne s'y agit pas d'un conflit inconscient dont l'angoisse-signal prépare une des névroses classiques.

Dans ces cas, l'angoisse est aussi le sentiment qui résulte du débordement du psychisme par l'affect. Mais il s'agit bien d'une violence destructrice. Aussi ce débordement ne s'explique pas par un refoulement qui délie la libido de ses représentations médiatrices vers l'action dans le monde et envers autrui. L'affect n'y est pas la libido à la recherche d'un objet. Il n'y a là tout simplement pas d'objet dont l'investissement conduirait la pulsion à l'apaisement. L'affect qui déborde est de l'ordre de la destruction et de la négation. Un jour un patient sujet à ces crises d'angoisse paroxysmale disait même verbalement: «il y a en moi une volonté violente de toujours nier et dénier ». À ce moment m'est revenu en mémoire ce que, dans le Faust de Goethe, l'esprit du pur mal 
dit par la bouche de Méphisto : «Ich bin der Geist der je verneint; je suis l'esprit qui toujours (dé)nie».

Ces énoncés rappellent étrangement la formule paradoxale par laquelle, à la fin de sa petite étude sur la Verneinung (dé-négation), Freud caractérise la schizophrénie : die allgemeine Verneinungslust, le plaisir généralisé de la (dé)négation. On pourrait approfondir ce trait de parenté entre les patients tourmentés par l'angoisse paroxysmale et les psychotiques, de même que la présence, dans les deux pathologies, des souffrances hypochondriaques.

\section{Concernant l'idée d'affect paroxysmal}

Le texte cité de 1929 atteste qu'en introduisant son concept de pulsion de mort, Freud a certainement cherché à donner un fondement pulsionnel à la violence paroxysmale. Szondi a ensuite proposé l'idée d'une pulsion paroxysmale, dont il reconnait une certaine parenté avec la pulsion freudienne de mort. Personnellement, je voudrais conserver l'idée szondienne de pulsion paroxysmale, mais je la comprends comme une vicissitude particulière de la pulsion libidinale. Selon moi, la «pulsion paroxysmale " est en réalité une figure de la transformation narcissique de la libido.

Je résume ma conception. Comme telle, la pulsion, étant poussée, comporte une agressivité plus ou moins violente. Cette violence est toujours une tendance de quelque manière destructrice, sans que la destruction soit par là le but de la pulsion. Gardonsnous, par conséquent, de déjà donner une qualification égoïque à ce premier moment de la formation pulsionnelle; a fortiori toute marque morale doit encore demeurer en suspens. Cette force de quelque manière destructrice de la poussée pulsionnelle prend une signification proprement psychologique et égoïque par la formation narcissique, et l'affirmation de soi et les oppositions et rivalités. Le sujet participatif-inflatif peut alors s'approprier narcissiquement la violence destructrice, afin de s'ériger dans sa puissance narcissique. Dans ce cas, la violence devient comme une puissance démoniaque à laquelle l'ego s'identifie et qui l'exalte dans la jouissance de la négativité. Le réfléchissement narcissique se déploie ensuite dans la mise en scène hystérique, pour soi-même, et de la destructivité et de l'angoisse. À ce moment la pulsion est devenue proprement paroxysmale. Le test szondien en atteste la présence dans le vecteur paroxysmal. Cela n'autorise toutefois pas la rétroprojection de la complexification historique de la pulsion, dont les données testologiques ne sont que l'indice indécis, dans l'idée d'une substance pulsionnelle spécifique.

\section{NOTES}

1.. Antoine Vergote a présenté cette étude - qui n'a pas encore été publiée - au Congrès international de Schicksalsanalyse (Analyse du Destin de Szondi, Cracovie, 15-18 août 1996). Professeur à Louvain et connu pour ses nombreux ouvrages, touchant à l'étude des présupposés du discours analytique, il vient de publier aux Éditions du Cerf De la psychanalyse à l'épreuve de la sublimation (1997). Le lecteur trouvera, dans ce livre, une 
excellente analyse des présupposés et des étroitesses philosophiques de Freud et une juste appréciation des découvertes de ce dernier. 